\title{
Novel Organophosphorus Cage Compound Produced by an Unexpected Oxidative Coupling of 1,2,4-Triphosphole: Crystal and Molecular Structures of Two Isomers of Formula $\mathrm{P}_{6} \mathrm{C}_{4} \mathrm{Bu}_{4}{ }_{4} \mathrm{CHSiMe}_{3}$
}

\author{
Maria Helena Araujo, ${ }^{a}$ Daniel A. Rajão, ${ }^{a}$ Antonio C. Doriguetto, ${ }^{b}$ Javier Ellena, ${ }^{b}$ Eduardo E. \\ Castellano, ${ }^{b}$ Peter B. Hitchcock ${ }^{c}$ and Vinicius Caliman ${ }^{*, a}$ \\ ${ }^{a}$ Departamento de Química, Universidade Federal de Minas Gerais, 31270-901 Belo Horizonte - MG, Brazil \\ ${ }^{b}$ Instituto de Física de São Carlos, Universidade de São Paulo, CP 369, 13560-970 São Carlos - SP, Brazil \\ ${ }^{c}$ School of Chemistry, Physics and Environmental Sciences, University of Sussex, Brighton, UK BNI 9QJ
}

$\mathrm{Li}\left(\mathrm{P}_{3} \mathrm{C}_{2} \mathrm{Bu}_{2}^{\mathrm{t}}\right)$ reage com $\mathrm{BrCH}\left(\mathrm{SiMe}_{3}\right)_{2}$, via adição oxidativa, formando um novo composto organofosforado de fórmula molecular $\mathrm{P}_{6} \mathrm{C}_{4} \mathrm{Bu}_{4}^{\mathrm{t}} \mathrm{CHSiMe}_{3}(\mathbf{1})$. O composto $\mathbf{1}$ foi caracterizado espectroscopicamente e sua estrutura molecular determinada através de difração de raios $\mathrm{X}$ de monocristais. Juntamente com $\mathbf{1}$, foi caracterizado outro composto (2) com a mesma fórmula molecular, mas apresentando uma estrutura completamente saturada.

Reaction of $\mathrm{Li}\left(\mathrm{P}_{3} \mathrm{C}_{2} \mathrm{Bu}_{2}^{\mathrm{t}}\right)$ with $\mathrm{BrCH}\left(\mathrm{SiMe}_{3}\right)_{2}$ results in a novel organophosphorus compound with the formula, $\mathrm{P}_{6} \mathrm{C}_{4} \mathrm{Bu}_{4}^{\mathrm{t}} \mathrm{CHSiMe}(\mathbf{1})$. Compound $\mathbf{1}$ was fully characterised spectroscopically and its unique molecular structure determined by single crystal X-ray diffraction. Another isomer (2) with a saturated structure was also fully characterised.

Keywords: organophosphorus compounds, multinuclear NMR, X-ray molecular structure

\section{Introduction}

The chemistry of organophosphorus compounds is in constant development, ${ }^{1}$ and it was the synthesis of $\mathrm{P} \equiv \mathrm{CBu}^{\mathrm{t}}$ in $1981,{ }^{2}$ the first stable phosphaalkyne at room temperature, that marked the beginning of this fascinating field of chemistry. This terbutylphosphaalkyne can undergo cyclo oligomerisation reactions to produce rings and cages. There are reports in the literature of compounds containing from 2 to $6 \mathrm{P} \equiv \mathrm{CBu}^{t}$ units with structures similar to those of the hydrocarbon analogues. ${ }^{3}$ The anionic rings $\left(\mathrm{P}_{3} \mathrm{C}_{2} \mathrm{Bu}_{2}^{\mathrm{t}}\right)^{-}$ (3) and $\left(\mathrm{P}_{2} \mathrm{C}_{3} \mathrm{Bu}_{3}^{\mathrm{t}}\right)^{-}$(4) have also been used as starting materials for the syntheses of several cages compounds containing phosphorus, e.g. $\mathrm{P}_{6} \mathrm{C}_{6} \mathrm{Bu}_{6}{ }_{6}, \mathrm{P}_{4} \mathrm{C}_{6} \mathrm{Bu}_{6} \mathrm{H}_{2}$, usually by oxidative coupling reactions using metal complexes ${ }^{3}$ and protonating reagents. ${ }^{4}$

Using phosphaalkyne, $\mathrm{P} \equiv \mathrm{CBu}^{\mathrm{t}}$, and an iron complex, $\left[\mathrm{Fe}\left(\eta^{6}-\mathrm{C}_{6} \mathrm{H}_{6}\right)\left(\eta^{4}-\mathrm{C}_{10} \mathrm{H}_{10}\right)\right]$, Zenneck and co-workers ${ }^{5}$ have prepared several organophosphorus compounds including $\mathrm{P}_{6} \mathrm{C}_{4} \mathrm{Bu}_{4}^{\mathrm{t}} \mathrm{CHBu}^{\mathrm{t}}(\mathbf{5})$ which contains two $\mathrm{P}_{3} \mathrm{C}_{2} \mathrm{Bu}_{2}^{\mathrm{t}}$ rings bonded together, and a $\mathrm{CHBu}^{t}$ group bridging two phosphorus atoms. Very recently, Avent et al. ${ }^{6}$ reported the synthesis of $\mathrm{P}_{6} \mathrm{C}_{4} \mathrm{Bu}_{4}^{\mathrm{t}} \mathrm{GeI}_{2}$ (6), whose structure is similar to that of compound 5 but with a $\mathrm{GeI}_{2}$ fragment at the bridging position.

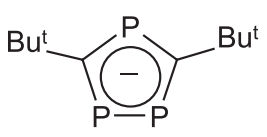

3

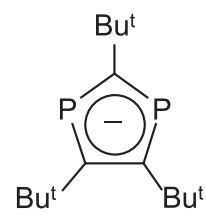

4



5

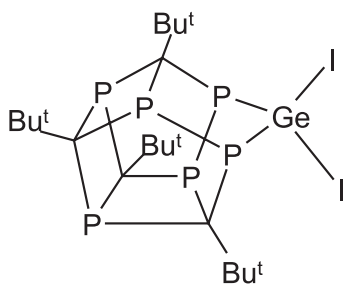

6

* e-mail: vcaliman@dedalus.lcc.ufmg.br 


\section{Results and Discussion}

We report herein another oxidative coupling reaction involving the anionic ring 3 to give a new unsaturated structure of formula $\mathrm{P}_{6} \mathrm{C}_{4} \mathrm{Bu}_{4}^{t} \mathrm{CHSiMe}_{3}(\mathbf{1})$. This new cage compound has an open structure with two $\mathrm{P}=\mathrm{C}$ double bonds and a $\mathrm{CHSiMe}_{3}$ group bridging two phosphorus atoms. Another isomer of this species, compound 2, was isolated from the same reaction mixture. This compound has a completely saturated structure, similar to that observed for compounds 5 and $\mathbf{6}$, but with a $\mathrm{CHSiMe}_{3}$ fragment at the bridging position, instead of $\mathrm{CHBu}^{t}$ and $\mathrm{GeI}_{2}$, respectively.

Compounds $\mathbf{1}$ and $\mathbf{2}$ were synthesised from the reaction of equimolar amounts of $\mathbf{3}$ with a bulky group ${ }^{7}$ $\mathrm{BrCH}\left(\mathrm{SiMe}_{3}\right)_{2}$, in DME. ${ }^{8}$ The mechanism of formation of both isomers is not clear, but one can speculate that it could take place via the well known 1,2,4-triphosphole $(7)^{9}$ which is also identified in the reaction mixture and probably dimerises to give $\mathbf{1}$ and $\mathbf{2}$ with loss of $\mathrm{CH}\left(\mathrm{SiMe}_{3}\right)_{3}$, which was not isolated. Compound $\mathbf{2}$ was also prepared in low yield (6.4\%) by Nixon and co-workers ${ }^{10}$ using a cobalt complex to promote the coupling.

Compounds $\mathbf{1}$ and $\mathbf{2}$, both air and moisture stable, were characterised by mass spectrometry, ${ }^{1} \mathrm{H},{ }^{13} \mathrm{C}$ and ${ }^{31} \mathrm{P}$ NMR spectroscopy and had their structures determined by single crystal X-ray diffraction studies.

The mass spectrum of $\mathbf{1}$ exhibits a molecular ion peak at $m / z, 548$, and the ${ }^{31} \mathrm{P}\left\{{ }^{1} \mathrm{H}\right\}$ NMR spectrum shows six resonances, two typical of $s p^{2}$ hybridised phosphorus with their chemical shifts in the high frequency region $(\delta 346.7$ and 294.7) and only small couplings indicating that no direct P-P bonds are present. ${ }^{11}$ The remaining resonances are all typical of $s p^{3}$ phosphorus $(\delta 74.6,45.6,30.0$ and -18.1). The resonance at $\delta 75.0$ shows two large coupling constants $\left({ }^{1} J_{\mathrm{P}(1) \mathrm{P}(2)} 268.2\right.$ and $\left.{ }^{1} J_{\mathrm{P}(1) \mathrm{P}(5)} 317.5 \mathrm{~Hz}\right)$ giving evidence that this phosphorus atom is bonded to other two in a P-P-P network.
The new tetracyclic structure of $\mathbf{1}$, presented in Figure 1 , was elucidated by single crystal $\mathrm{X}$-ray analysis ${ }^{12}$ and reveals three phosphorus atoms bonded together, $\mathrm{P}(2)-\mathrm{P}(1)$ $\mathrm{P}(5)$, and two $\mathrm{P}=\mathrm{C}$ doubled bonds. The distance between $\mathrm{P}(2)-\mathrm{P}(1)$ and $\mathrm{P}(1)-\mathrm{P}(5)$ (2.250(1) and 2.191(2) , respectively) are in the expected range for a $\mathrm{P}-\mathrm{P}$ single bond. ${ }^{13}$ The two doubled bonds $\mathrm{P}(3)-\mathrm{C}(3)$ and $\mathrm{P}(4)-\mathrm{C}(5)$ (1.668(4) and 1.676(4) A, respectively) are the shortest ones in this cage framework and lie in the expected range previously reported $(1.64-1.69 \AA) .{ }^{14}$ The $\mathrm{C}(3)$ and $\mathrm{C}(5)$ carbons show bond angles typical of $s p^{2}$-hybridised atoms.

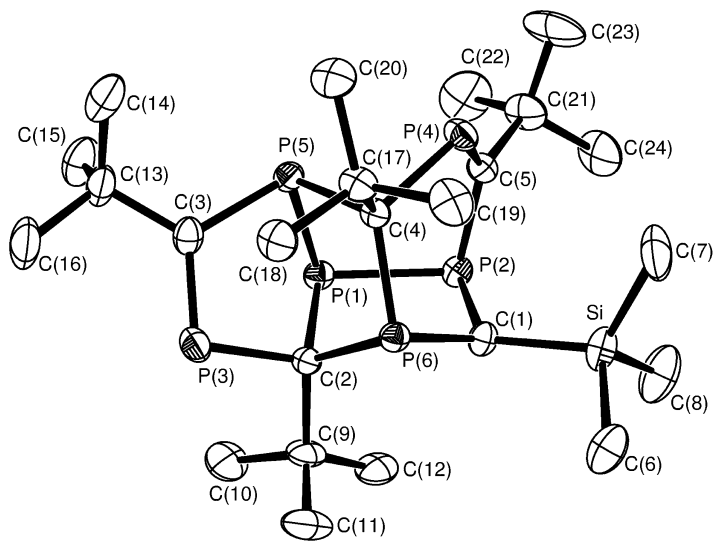

Figure 1. Molecular structure of $\mathrm{P}_{6} \mathrm{C}_{4} \mathrm{Bu}_{4}^{\mathrm{t}} \mathrm{CHSiMe}_{3}$ (1), showing the atoms labelling (hydrogen atoms are omitted for clarity).

Isomer 2 with the same MS molecular ion $\mathrm{m} / \mathrm{z} 548$ was characterised by ${ }^{31} \mathrm{P}\left\{{ }^{1} \mathrm{H}\right\}$ NMR spectroscopy showing six resonances all typical of $s p^{3}$ hybridised phosphorus. The two resonances at $\delta 196.9$ and 178.4 with small P-P couplings (in the $15-30 \mathrm{~Hz}$ range) indicate the presence of two phosphorus bonded only to carbon atoms. ${ }^{15}$ Large P-P couplings for the remaining four resonances $\left({ }^{1} J_{\mathrm{P}(1) \mathrm{P}(2)} 228.4\right.$ and ${ }^{1} J_{\mathrm{P}(4) \mathrm{P}(5)} 237.2 \mathrm{~Hz}$ ) suggest four phosphorus atoms bonded together two by two.

The molecular structure of $\mathbf{2}$ is shown in Figure $2 .{ }^{16} \mathrm{It}$ presents the two five membered rings joined side by side
2<smiles>CC(C)CC(C)C</smiles>

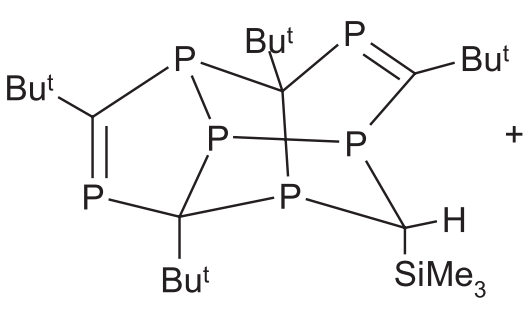

1

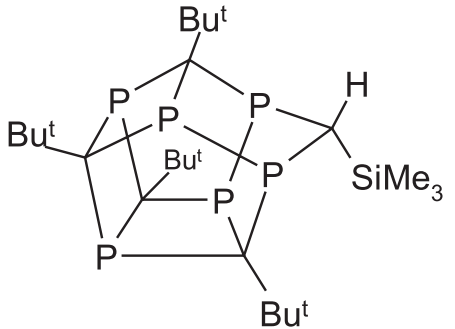

2

Scheme 1. 
Table 1. Selected bond lengths and angles for $\mathrm{P}_{6} \mathrm{C}_{4} \mathrm{Bu}_{4}^{\mathrm{t}} \mathrm{CHSiMe}_{3}(\mathbf{1})$

\begin{tabular}{|c|c|c|c|c|c|}
\hline \multicolumn{6}{|c|}{ Bond lengths ( $($ ) } \\
\hline $\mathrm{P}(1)-\mathrm{P}(2)$ & $2.251(1)$ & $\mathrm{P}(1)-\mathrm{P}(5)$ & $2.190(2)$ & $\mathrm{P}(1)-\mathrm{C}(2)$ & $1.887(4)$ \\
\hline$P(2)-C(1)$ & $1.868(4)$ & $\mathrm{P}(2)-\mathrm{C}(5)$ & $1.834(4)$ & $P(3)-C(2)$ & $1.872(4)$ \\
\hline $\mathrm{P}(3)-\mathrm{C}(3)$ & $1.669(4)$ & $\mathrm{P}(4)-\mathrm{C}(4)$ & $1.881(3)$ & $P(4)-C(5)$ & $1.678(4)$ \\
\hline$P(5)-C(3)$ & $1.848(4)$ & $\mathrm{P}(5)-\mathrm{C}(4)$ & $1.937(4)$ & $\mathrm{P}(6)-\mathrm{C}(1)$ & $1.821(4)$ \\
\hline $\mathrm{P}(6)-\mathrm{C}(2)$ & $1.903(4)$ & $\mathrm{P}(6)-\mathrm{C}(4)$ & $1.891(3)$ & $\mathrm{C}(1)-\mathrm{Si}$ & $1.896(4)$ \\
\hline \multicolumn{6}{|c|}{ Bond angles $\left({ }^{\circ}\right)$} \\
\hline $\mathrm{P}(2)-\mathrm{P}(1)-\mathrm{P}(5)$ & $102.18(5)$ & & & $\mathrm{P}(2)-\mathrm{P}(1)-\mathrm{C}(2)$ & $97.80(12)$ \\
\hline $\mathrm{P}(5)-\mathrm{P}(1)-\mathrm{C}(2)$ & $89.87(12)$ & & & $\mathrm{C}(1)-\mathrm{P}(2)-\mathrm{C}(5)$ & $99.5(2)$ \\
\hline $\mathrm{C}(2)-\mathrm{P}(3)-\mathrm{C}(3)$ & $100.7(2)$ & & & $\mathrm{C}(4)-\mathrm{P}(4)-\mathrm{C}(5)$ & $107.8(2)$ \\
\hline $\mathrm{C}(1)-\mathrm{P}(6)-\mathrm{C}(2)$ & $96.0(2)$ & & & $\mathrm{P}(3)-\mathrm{C}(3)-\mathrm{P}(5)$ & $117.3(2)$ \\
\hline$P(3)-C(3)-C(13)$ & $123.1(3)$ & & & $P(5)-C(3)-C(13)$ & $119.5(3)$ \\
\hline$P(2)-C(5)-P(4)$ & $122.5(2)$ & & & $P(2)-C(5)-C(21)$ & $116.6(3)$ \\
\hline
\end{tabular}

by a $\mathrm{CHSiMe}_{3}$ bridge forming a cage with four fivemembered and three four-membered rings. The average $\mathrm{C}$ $\mathrm{C}$ and P-C distances are 1.54(2) and 1.88(1) $\AA$, respectively, which are in the expected range. ${ }^{13,14}$ The $\mathrm{P}(2)-\mathrm{P}(1)$ and $\mathrm{P}(4)$ -

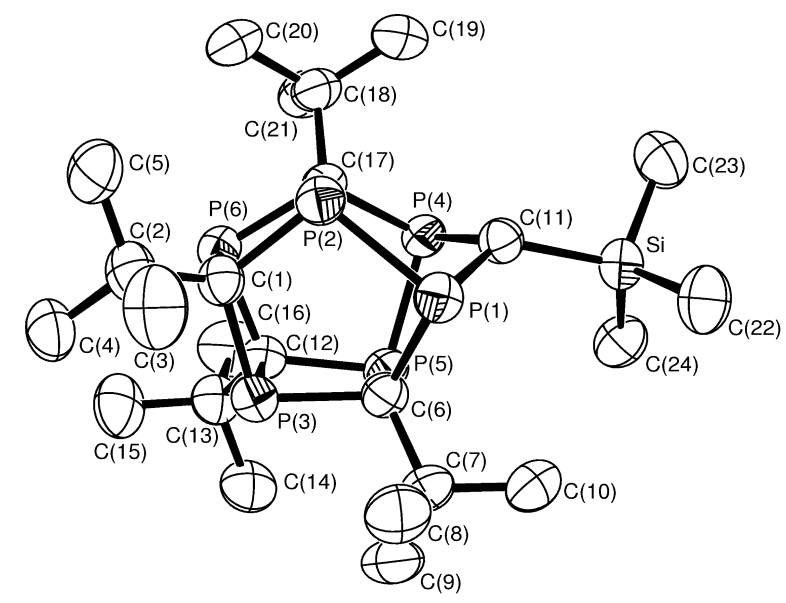

Figure 2. Molecular structure of $\mathrm{P}_{6} \mathrm{C}_{4} \mathrm{Bu}_{4}^{t} \mathrm{CHSiMe}_{3}$ (2), showing the atoms labelling (hydrogen atoms are omitted for clarity).
P(5) bond distances are 2.201(1) and 2.190(1) $\AA$, respectively, in agreement with the triad, P-P-P, found in structure 1.

\section{Acknowledgements}

This work has been supported by Brazilian agencies CNPq, FAPEMIG and FAPESP (Projeto Temático No. 98/ 12151-1). J.E. and A.C.D. thank FAPESP and CNPq, respectively, for the postdoctoral fellowship. We also thank Prof. J. F. Nixon (UK) for his constant encouragement.

\section{Supplementary Material}

Crystallographic data (excluding structure factors) for the structures in this paper have been deposited with the Cambridge Crystallographic Data Centre as supplementary publication numbers CCDC 173911 and 173912. Copies of the data can be obtained, free of charge, on apllication to CCDC, 12 Union Road, Cambridge CB2 1EZ, UK (fax:+44 1223336033 or e-mail: deposit@ccdc.cam.ac.uk).

Table 2. Selected bond lengths and angles for $\mathrm{P}_{6} \mathrm{C}_{4} \mathrm{Bu}_{4}^{\mathrm{t}} \mathrm{CHSiMe}_{3}$ (2)

\begin{tabular}{|c|c|c|c|c|c|}
\hline \multicolumn{6}{|c|}{ Bond lengths $(\AA)$} \\
\hline $\mathrm{P}(1)-\mathrm{P}(2)$ & $2.201(1)$ & $\mathrm{P}(4)-\mathrm{P}(5)$ & $2.190(1)$ & $\mathrm{P}(1)-\mathrm{C}(6)$ & $1.883(3)$ \\
\hline $\mathrm{P}(1)-\mathrm{C}(11)$ & $1.874(3)$ & $P(2)-C(1)$ & $1.868(3)$ & $\mathrm{P}(2)-\mathrm{C}(17)$ & $1.906(3)$ \\
\hline $\mathrm{P}(3)-\mathrm{C}(1)$ & $1.876(3)$ & $\mathrm{P}(3)-\mathrm{C}(12)$ & $1.893(3)$ & $\mathrm{P}(3)-\mathrm{C}(6)$ & $1.888(3)$ \\
\hline $\mathrm{P}(4)-\mathrm{C}(11)$ & $1.860(3)$ & $P(4)-C(17)$ & $1.874(3)$ & $\mathrm{P}(5)-\mathrm{C}(12)$ & $1.880(3)$ \\
\hline$P(5)-C(6)$ & $1.907(3)$ & $\mathrm{P}(6)-\mathrm{C}(1)$ & $1.901(3)$ & $P(6)-C(12)$ & $1.880(3)$ \\
\hline$P(6)-C(17)$ & $1.882(3)$ & $\mathrm{Si}-\mathrm{C}(11)$ & $1.911(3)$ & & \\
\hline \multicolumn{6}{|c|}{ Bond angles $\left({ }^{\circ}\right)$} \\
\hline $\mathrm{P}(1)-\mathrm{C}(11)-\mathrm{Si}$ & $125.9(2)$ & & & $\mathrm{P}(4)-\mathrm{C}(11)-\mathrm{P}(1)$ & $108.0(2)$ \\
\hline $\mathrm{P}(4)-\mathrm{C}(11)-\mathrm{Si}$ & $111.6(2)$ & & & $\mathrm{C}(6)-\mathrm{P}(1)-\mathrm{C}(11)$ & $101.86(2)$ \\
\hline $\mathrm{C}(6)-\mathrm{P}(1)-\mathrm{P}(2)$ & $98.98(3)$ & & & $\mathrm{C}(11)-\mathrm{P}(1)-\mathrm{P}(2)$ & $91.70(2)$ \\
\hline $\mathrm{C}(1)-\mathrm{P}(2)-\mathrm{C}(17)$ & $87.78(2)$ & & & $\mathrm{C}(1)-\mathrm{P}(2)-\mathrm{P}(1)$ & $101.13(4)$ \\
\hline $\mathrm{C}(17)-\mathrm{P}(2)-\mathrm{P}(1)$ & $100.82(5)$ & & & $\mathrm{C}(1)-\mathrm{P}(3)-\mathrm{C}(6)$ & $102.77(7)$ \\
\hline $\mathrm{C}(1)-\mathrm{P}(3)-\mathrm{C}(12)$ & $87.37(2)$ & & & $\mathrm{C}(6)-\mathrm{P}(3)-\mathrm{C}(12)$ & $88.02(2)$ \\
\hline
\end{tabular}




\section{References}

1. Dillon, K. B.; Matthey, F.; Nixon, J. F.; Phosphorus: The Carbon Copy, John Wiley: Chichester, 1998, p. 266 and references therein.

2. Becker, G.; Gresser, G.; Uhl, W.; Z. Naturforsch., B 1981, 36, 16.

3. Streubel, R.; Angew. Chem., Int. Ed. Engl. 1995, 34, 436; Caliman, V.; Hitchcock, P. B.; Nixon, J. F.; Hofmann, M.; Schleyer, P. R.; Angew. Chem., Int. Ed. Engl. 1994, 33, 2202; Geissler, B.; Wettling, T.; Barth, S.; Binger, P.; Regitz, M.; Synthesis 1994, 1337; Tabellion, F.; Nachbauer, A.; Leininger, S.; Peters, C.; Preuss, F.; Regitz, M.; Angew. Chem., Int. Ed. Engl. 1998, 37, 1233.

4. Caliman, V.; Quim. Nova 2000, 23, 346.

5. Hu, D.; Schäufele, H.; Pritzkow, H.; Zenneck, U.; Angew. Chem., Int. Ed. Engl. 1989, 28, 900.

6. Avent, A. G.; Cloke, F. G. N.; Francis, M. D.; Hitchcock, P. B.; Nixon, J. F.; Chem. Commun. 2000, 879.

7. Lappert, M. F.; Liu, D. S.; J. Organomet. Chem. 1995, 500, 203.

8. General procedure for the preparation of $\mathrm{P}_{6} \mathrm{C}_{4} \mathrm{Bu}_{4}^{t} \mathrm{CHSiMe}_{3} \mathbf{1}$ and 2: $\mathrm{HCBr}\left(\mathrm{SiMe}_{3}\right)_{2}(0.6 \mathrm{~mL}, 2.31 \mathrm{mmol})$ in $\mathrm{DME}(10 \mathrm{~mL})$, was added slowly to a solution of $\mathrm{Li}\left(\mathrm{P}_{3} \mathrm{C}_{2} \mathrm{Bu}_{2}^{\mathrm{t}}\right)(2.31 \mathrm{mmol})$ in $15 \mathrm{~mL}$ of DME, at $-30{ }^{\circ} \mathrm{C}$ and the resulting mixture was stirred for $20 \mathrm{~h}$. The $\mathrm{LiBr}$ formed during the reaction was filtered off and the solvent evaporated under vacuum. The residue was purified by column chromatography (kieselgel/hexane) to give two yellow bands. The first was characterised as compound $\mathbf{1}$ (250 mg, 20\%), and the second as compound 2 (200 mg, $16 \%)$. Recrystalisation from hexane at room temperature afforded yellow crystals suitable for X-ray diffraction study for both compounds.

9. Caliman, V.; Hitchcock, P. B.; Nixon, J. F.; Heteroatom Chem. 1998, $9,1$.

10. Hitchcock, P. B.; Nixon, J. F.; Buyukkidan, N. S.; Chem. Commun. 2001, 2720.

11. Compound 1: ${ }^{31} \mathrm{P}\left\{{ }^{1} \mathrm{H}\right\}$ NMR (121.44 MHz, $\mathrm{C}_{6} \mathrm{D}_{6}, \mathrm{H}_{3} \mathrm{PO}_{4}$ ext. standard, $\left.25{ }^{\circ} \mathrm{C}\right): \delta 346.7[\mathrm{P}(3)], 294.7[\mathrm{P}(4)], 74.6[\mathrm{P}(1)]$, $45.6[\mathrm{P}(5)], 30.0[\mathrm{P}(6)],-18.1[\mathrm{P}(2)] ;{ }^{1} J_{\mathrm{P}(1) \mathrm{P}(5)} 317.5 \mathrm{~Hz},{ }^{1} J_{\mathrm{P}(1) \mathrm{P}(2)}$ $268.2 \mathrm{~Hz} .{ }^{13} \mathrm{C}\left\{{ }^{1} \mathrm{H}\right\}$ NMR $\left(75.43 \mathrm{MHz}, \mathrm{C}_{6} \mathrm{D}_{6}, 25^{\circ} \mathrm{C}\right): \delta 204.2$ (m, PCP); 200.52 (m, PCP); 91.89 (m, P=CP); 75.44 (m, $\mathrm{P}=\mathrm{CP}) ; 43.06\left(\mathrm{~m}, \mathrm{C}\left(\mathrm{CH}_{3}\right)_{3}\right) ; 42.21\left(\mathrm{~m}, \mathrm{C}\left(\mathrm{CH}_{3}\right)_{3}\right) ; 40.65(\mathrm{~m}$, $\left.\mathrm{C}\left(\mathrm{CH}_{3}\right)_{3}\right) ; 39.23\left(\mathrm{~m}, \mathrm{C}\left(\mathrm{CH}_{3}\right)_{3}\right) ; 36.80(\mathrm{~m}, \mathrm{CH}) ; 32.70(\mathrm{~m}$, $\left.\mathrm{C}\left(\mathrm{CH}_{3}\right)_{3}\right) ; 30.39\left(\mathrm{~m}, \mathrm{C}\left(\mathrm{CH}_{3}\right)_{3}\right) ; 29.13\left(\mathrm{~m}, \mathrm{C}\left(\mathrm{CH}_{3}\right)_{3}\right) ; 0.03$ (s, $\left.\mathrm{Si}\left(\mathrm{CH}_{3}\right)_{3}\right) .{ }^{1} \mathrm{H}$ NMR (300.00 MHz, $\left.\mathrm{C}_{6} \mathrm{D}_{6}\right): \delta 2.46(\mathrm{~m}, \mathrm{CH})$; $1.53\left(\mathrm{~s}, 18 \mathrm{H}, 2 \mathrm{C}\left(\mathrm{CH}_{3}\right)_{3}\right) ; 1.36\left(\mathrm{~d}, 9 \mathrm{H}, \mathrm{C}\left(\mathrm{CH}_{3}\right)_{3},{ }^{4} J_{(\mathrm{HP})} 2.14\right.$
$\mathrm{Hz}) ; 1.30\left(\mathrm{~d}, 9 \mathrm{H}, \mathrm{C}\left(\mathrm{CH}_{3}\right)_{3},{ }^{4} J_{(\mathrm{HP})} 1.4 \mathrm{~Hz}\right) ; 0.22\left(\mathrm{~s}, 9 \mathrm{H}, \mathrm{Si}\left(\mathrm{CH}_{3}\right)_{3}\right)$. EI-MS m/z (\%): 548 (30) $\left[\mathrm{P}_{6} \mathrm{C}_{4} \mathrm{Bu}_{4}^{\mathrm{t}} \mathrm{CHSiMe}_{3}\right]^{+}, 533$ (12) $\left[\mathrm{P}_{6} \mathrm{C}_{4} \mathrm{Bu}_{4}^{\mathrm{t}} \mathrm{CHSiMe}_{2}\right]^{+}, 491$ (11) $\left[\mathrm{P}_{6} \mathrm{C}_{4} \mathrm{Bu}_{3}^{\mathrm{t}} \mathrm{CHSiMe}_{3}\right]^{+}, 476$ (13) $\left[\mathrm{P}_{6} \mathrm{C}_{4} \mathrm{Bu}_{4}^{\mathrm{t}} \mathrm{CH}\right]^{+}$.

12. Crystal data for 1 : $\mathrm{P}_{6} \mathrm{C}_{24} \mathrm{H}_{46} \mathrm{Si}, M=548.51$, monoclinic $P 2_{1} / \mathrm{n}$ (№ 14), $a=11.768$ (3), $b=15.249$ (6), $c=17.033$ (5) $\AA, \mathrm{b}=$ 93.35(2), $V=3051(2) \AA^{3}, \mathrm{~T}=173(2) \mathrm{K}, Z=4, \mu=0.405 \mathrm{~mm}^{-1}$, $\lambda=0.71073 \AA$, 5608 reflections collected, 5339 independent $\left(\mathrm{R}_{\text {int }}=0.030\right), 3901$ reflections with $\mathrm{I}>2 \sigma(\mathrm{I}) \mathrm{R}(\mathrm{F})=0.049$, $w R\left(F^{2}\right)=0.108$ for $\mathrm{I}>2 \sigma \mathrm{I}, \mathrm{R}(\mathrm{F})=0.078, \mathrm{wR}\left(\mathrm{F}^{2}\right)=0.123$ for all data.

13. Maigrot, N.; Ricard, L.; Charrier, C.; Golf, P. L.; Mathey, F.; Bull. Soc. Chim. Fr. 1992, 129, 76; Bevierre, M. O.; Mercier, F.; Ricard, L.; Mathey, F.; Bull. Soc. Chim. Fr. 1992, 129, 1; Bartsch, R.; Hitchcock, P. B.; Nixon, J. F.; J. Chem. Soc., Chem. Commun. 1989, 1046.

14. Geissler, B.; Barth, S.; Bergsträsser, U.; Slany, M.; Durkin, J.; Hitchcock, P. B.; Hofmann, M.; Binger, P.; Nixon, J. F.; Schleyer, P. R.; Regitz, M.; Angew. Chem., Int. Ed. Engl. 1995, 34, 484; Gudat, D.; Niecke, E.; Arif, A. M.; Cowley, A. H.; Quashie, S.; Organometallics 1986, 5, 593; Kroto, H. W.; Nixon, J. F.; Ohashi, O.; Ohno, K.; Simmons, N. P.; J. Mol. Spectrosc. 1984, 103, 113.

15. Compound 2: ${ }^{31} \mathrm{P}\left\{{ }^{1} \mathrm{H}\right\}$ NMR (161.97 $\mathrm{MHz}, \mathrm{CDCl}_{3}, \mathrm{H}_{3} \mathrm{PO}_{4}$ ext. standard, $\left.25^{\circ} \mathrm{C}\right): \delta 196.9[\mathrm{P}(3)], 178.4[\mathrm{P}(6)], 148.5$ $[\mathrm{P}(2)], 139.3[\mathrm{P}(5)], 122.9[\mathrm{P}(1)], 120.5[\mathrm{P}(4)] ;{ }^{1} J_{\mathrm{P}(4) \mathrm{P}(5)} 237.2$ $\mathrm{Hz},{ }^{1} \mathrm{~J}_{\mathrm{P}(1) \mathrm{P}(2)} 228.4 \mathrm{~Hz} .{ }^{13} \mathrm{C}\left\{{ }^{1} \mathrm{H}\right\} \mathrm{NMR}\left(100.61 \mathrm{MHz}, \mathrm{CDCl}_{3}\right.$, $\left.25^{\circ} \mathrm{C}\right): \delta 41.5\left(\mathrm{~s}, \mathrm{CMe}_{3}\right) ; 41.1\left(\mathrm{~s}, 2\left(\mathrm{CMe}_{3}\right)\right) ; 40.6\left(\mathrm{~s}, C \mathrm{Me}_{3}\right)$; $37.1\left(\mathrm{~m}, C \mathrm{Bu}^{\mathrm{t}}\right) ; 35.1\left(\mathrm{~m}, C \mathrm{Bu}^{\mathrm{t}}\right) ; 30.3\left(\mathrm{~m}, C \mathrm{Bu}^{\mathrm{t}}\right) ; 29.0(\mathrm{~m}$, $\left.\mathrm{CBu}^{\mathrm{t}}\right) ; 26.7\left(\mathrm{~m}, \mathrm{CSiMe}_{3}\right) ; 1.1\left(\mathrm{~s}, \mathrm{CH}_{3}\right) .{ }^{1} \mathrm{H} \mathrm{NMR}(400.13 \mathrm{MHz}$, $\left.\mathrm{CDCl}_{3}\right): \delta 1.62(\mathrm{~m}, \mathrm{CH}) ; 1.27\left(\mathrm{~s}, 9 \mathrm{H},\left(\mathrm{CH}_{3}\right)_{3}\right) ; 1.23(\mathrm{~s}, 9 \mathrm{H}$, $\left.\left(\mathrm{CH}_{3}\right)_{3}\right) ; 1.17\left(\mathrm{~s}, 9 \mathrm{H},\left(\mathrm{CH}_{3}\right)_{3}\right) ; 1.16\left(\mathrm{~s}, 9 \mathrm{H},\left(\mathrm{CH}_{3}\right)_{3}\right) ; 0.26(\mathrm{~s}$, $\left.9 \mathrm{H}, \quad \mathrm{Si}\left(\mathrm{CH}_{3}\right)_{3}\right) . \quad$ EI-MS $\mathrm{m} / z \quad(\%): 548 \quad$ (100) $\left[\mathrm{P}_{6} \mathrm{C}_{4} \mathrm{Bu}_{4}^{\mathrm{t}} \mathrm{CHSiMe}_{3}\right]^{+}, 491$ (35) $\left[\mathrm{P}_{6} \mathrm{C}_{4} \mathrm{Bu}_{3}^{\mathrm{t}} \mathrm{CHSiMe}_{3}\right]^{+}, 169$ (17) $\left[\mathrm{CBu}^{\mathrm{t}}\right]^{+}, 73(70)\left[\mathrm{SiMe}_{3}\right]^{+}$.

16. Crystal data for 2: $\mathrm{P}_{6} \mathrm{C}_{24} \mathrm{H}_{46} \mathrm{Si}, M=548.51$, monoclinic $\mathrm{P}_{1} / \mathrm{c}$ (standard № 14), a = 12.9500(2), b = 19.4710(3), c = 12.0920(2) $\AA, \mathrm{b}=95.625(1)^{\circ}, \mathrm{V}=3034.31(8) \AA^{3}, \mathrm{~T}=173(2)$, $\mathrm{Z}=4, \mu=0.405 \mathrm{~mm}^{-1}, \lambda=0.71070 \AA$, 60382 measured reflections, 5334 independent reflections $\left(\mathrm{R}_{\mathrm{int}}=0.0858\right), 4488$ reflections with $\mathrm{I}>2 \sigma(\mathrm{I}), \mathrm{R}(\mathrm{F})=0.0446, \mathrm{wR}\left(\mathrm{F}^{2}\right)=0.1366$ for $\mathrm{I}>2 \sigma \mathrm{I}, \mathrm{R}(\mathrm{F})=0.0654, \mathrm{wR}\left(\mathrm{F}^{2}\right)=0.1662$ for all data.

Received: February 22, 2002

Published on the web: August 6, 2002

FAPESP helped in meeting the publication costs of this article. 